\title{
AVALIAÇÃO DO ATENDIMENTO AOS DIAGNÓSTICOS DE HIPERTENSÃO ARTERIAL E DIABETES MELLITUS NA ATENÇÃO BÁSICA DE SAÚDE EM MUNICÍPIO DO INTERIOR PARAIBANO
}

\section{EVALUATION OF THE SERVICE FOR DIAGNOSIS OF ARTERIAL HYPERTENSION AND DIABETES MELLITUS IN BASIC HEALTH CARE IN A CITY IN THE COUNTRYSIDE OF PARAÍBA}

Luana Lindolfo Querino ${ }^{1}$ Alexsandra Laurindo Leite ${ }^{2}$ Maria Iranilda Silva Magalhães ${ }^{3}$ Maria Alciene Saraiva de Souza ${ }^{4}$ Margarida Maria Lima Rolim de Oliveira ${ }^{5}$ Jessica Alves Moreira ${ }^{6}$

RESUMO: Objetivo: Avaliar o atendimento aos diagnósticos de Hipertensão Arterial e Diabetes Mellitus na Atenção Básica de Saúde em Município do interior paraibano. Método: Trata-se de um estudo exploratório, descritivo e quantitativo, que foi realizado na Unidade Básica de Saúde José Nilson de Sousa da cidade Cajazeiras$\mathrm{PB}$, através de um questionário para 30 usuários do serviço, portadores de Diabetes

\footnotetext{
${ }^{1}$ Acadêmica do Curso de Bacharelado em Biomedicina da Faculdade Santa Maria (FSM), CajazeirasPB. E-mail: luannacz@hotmail.com.

${ }^{2}$ Especialista em Hematologia Clinica pelo Instituto Leão Sampaio Ensino Universitário. Especialista em Citologia pelo Instituto Leão Sampaio Ensino Universitário. Biomédica formada pelo Instituto Leão Sampaio Ensino Universitário. Docente do Curso de Bacharelado em Biomedicina da Faculdade Santa Maria - FSM, Cajazeiras-PB. E-mail: alexsandralaurindo@gmail.com.

${ }^{3}$ Doutoranda do Programa de Pós Graduação de Pesquisa e Inovação na Faculdade de Medicina do ABC Paulista. Mestre em Ciências da Saúde pela Universidade Cruzeiro do Sul. Especialista em Ciências da Educação, Biologia e Psicopedagogia. Biomédica formada pelo Instituto Leão Sampaio Ensino Universitário. E-mail: iranildamagalhaes@gmail.com.

${ }^{4}$ Mestranda em Ciências da Saúde pela Faculdade de Medicina do ABC Paulista. Especialista em Educação, Desenvolvimento e Políticas Públicas educacionais. Biomédica formada pelo Instituto Leão Sampaio Ensino Universitário. E-mail: alciene_ss@yahoo.com.

${ }^{5}$ Especialista em Saúde da Família pela Universidade Federal da Paraíba - UFPB. Enfermeira formada pela Escola de Enfermagem Santa Emília do Rodat. Docente do Curso de Bacharelado em Enfermagem da Faculdade Santa Maria (FSM), Cajazeiras-PB. E-mail: mmlroliveira@hotmail.com.

${ }^{6}$ Especialista em Hematologia Clinica pelo Instituto Leão Sampaio Ensino Universitário. Especialista em Docência do Ensino Superior pela Faculdade Santa Maria - FSM, Cajazeiras-PB. Biomédica formada pelo Instituto Leão Sampaio Ensino Universitário. Docente do Curso de Bacharelado em Biomedicina da Faculdade Santa Maria - FSM, Cajazeiras-PB. E-mail: jessica.alvesmoreira@hotmail.com.
} 
Mellitus e Hipertensão Arterial Sistêmica, com idade entre 34 e 95 anos, contendo questões acerca do tema exposto. A coleta de dados foi feita a partir da aprovação Comitê de Ética em Pesquisa (CEP) e seguiu de acordo com as normas da resolução 466/12 do Conselho Nacional de Saúde. Resultados: Em relação à classificação socioeconômica, foi observado que o maior percentual dos participantes era casado $15(50 \%)$. O nível de escolaridade que predominou foi o Ensino Fundamental Incompleto com o total de 21 (70\%), e a maioria dos entrevistados possui, renda familiar de 1 salário mínimo com 15 (50\%). Houve prevalência variando de 34 a 95 anos de idade, com predominância do sexo feminino 22 (73\%). Na frequência de realização de exames, prevaleceu o percentual de $37 \%$ com exames realizados em 2018. Quanto ao percentual de entrevistados que foram encaminhados para outros profissionais de saúde, o estudo mostra que a maioria dos usuários foi encaminhada apenas para o cardiologista. Conclusão: As análises confirmaram que as ações desenvolvidas pela UBS não estão de acordo com o Plano de Reorganização da Atenção à Hipertensão Arterial e ao Diabetes Mellitus apresentado pelo Governo Federal através do Ministério da Saúde. Os usuários do SUS não são instruídos pelo médico/enfermeiro da UBS a procurarem outros profissionais da saúde que compõem a equipe multidisciplinar, tornando necessário um maior empenho da equipe da UBS na assistência aos cadastrados no programa Hirperdia.

Descritores: Hipertensão Arterial; Diabetes Mellitus; Atenção Básica de Saúde.

ABSTRACT: Objective: To evaluate the service for diagnosis of Hypertension and Diabetes Mellitus in Basic Health Care in a city in the countryside of Paraiba. Method: This was an exploratory, descriptive and quantitative study carried out at the José Nilson de Sousa Basic Health Unit of Cajazeiras-PB, through a questionnaire for 30 users of the service, with Diabetes Mellitus and Systemic Arterial Hypertension, aged between 34 and 95 years, with questions about the subject of interest. Data collection occurred after approval by the Research Ethics Committee (REC) and followed the norms of Resolution 466/12 of the National Health Council. Results: Regarding socioeconomic classification, the highest percentage of the participants was married, 15 (50\%). The predominant level of schooling was Incomplete Elementary School, with 21 (70\%), and the majority of the interviewees had a family income of one minimum wage, with 15 (50\%). The predominant age range was from 34 to 95 years, predominantly female 22 (73\%). In the frequency of tests, $37 \%$ underwent tests in 2018. As for the percentage of interviewees who were referred to other health professionals, the study shows that most users were referred only to the cardiologist. Conclusion: The analyses confirmed that the actions developed by the BHU are not in accordance with the Health Care Reorganization Plan to Hypertension and Diabetes Mellitus presented by the Federal Government through the Ministry of Health. UHS users are not instructed by the BHU doctor/nurse to look for other health professionals who make up the multidisciplinary team, which requires a greater commitment of the $\mathrm{BHU}$ team in the assistance to those enrolled in the Hirperdia program.

Descriptors: Arterial Hypertension; Diabetes Mellitus; Basic Health Care. 


\section{INTRODUÇÃO}

As Doenças Crônicas Não Transmissíveis são patologias derivadas de vários fatores de riscos ambientais, comportamentais e até mesmo de fatores genéticos, sendo caracterizadas como não infecciosas e irreversíveis, de duração prolongada e com longo período de latência, podendo gerar incapacidade funcional. A maior incidência das DCNT está na população adulta e particularmente entre os idosos de modo mais acentuado (LOPES; REZENDE; CLABRIA, 2017).

Sendo consideradas as mais comuns nos dias atuais e um grave problema de saúde pública no Brasil e no mundo, podem-se observar uma grande incidência e prevalência da Hipertensão Arterial Sistêmica e da Diabetes Mellitus, que são Doenças Crônicas Não Transmissíveis (DCNT). Embora sejam realizadas ações e criados vários programas de promoção, prevenção e recuperação da saúde pelos órgãos federais, estaduais e municipais (FERNANDEZ; POLLARO; GONÇALVES, 2016).

Devido ao crescimento e envelhecimento populacional, um crescimento urbanizado e a adesão de estilo de vida, o sedentarismo, dieta inadequada propiciando a obesidade, é notório o aumento da incidência e prevalência da diabetes em todo o mundo (CORTEZ et al., 2015).

O Diabetes Mellitus (DM) é definido por uma hiperglicemia, uma vez que os distúrbios no metabolismo de gorduras, proteínas e carboidratos, é manifestado por uma ausência ou uma produção insuficiente de insulina, ou mesmo por uma incapacidade de as células reconhecerem a insulina, causando um transtorno metabólico onde o hormônio não permite a entrada da glicose na célula (PEREIRA; FIGUEIREDO, 2017).

A Hipertensão Arterial Sistêmica (HAS) é uma condição clínica multifatorial, caracterizada por níveis elevados e sustentados de pressão arterial - PA (PA $\geq 140 x$ $90 \mathrm{mmHg}$ ), associada a alterações funcionais e/ou estruturais dos órgãos-alvo: 
coração, encéfalo, rins e vasos sanguíneos e alterações metabólicas, com aumento do risco de eventos cardiovasculares fatais e não fatais (BRASIL, 2013).

A HAS, além de ser causa direta de cardiopatia hipertensiva, é fator de risco para doenças decorrentes de aterosclerose e trombose, que se manifestam, predominantemente, por doença isquêmica cardíaca, cerebrovascular, vascular periférica e renal, manifestando, também, como fator etiológico da insuficiência cardíaca. Déficits cognitivos, como doença de Alzheimer e demência vascular, também têm a doença em fases mais precoces da vida como fator de risco. Essa quantidade de influências coloca a HAS na origem de muitas DCNT e, portanto, caracteriza-a como uma das causas de maior redução da expectativa e da qualidade de vida dos indivíduos (BRASIL, 2013).

Atenção Básica foi criada com o objetivo de ser o primeiro nível de atenção em saúde da população, abrangendo a promoção, prevenção, diagnóstico, tratamento, reabilitação, redução de danos e a manutenção da saúde; trabalho realizado nas Unidades Básicas de Saúde (UBS), composta por uma equipe multiprofissional denominada Estratégia de Saúde da Família, proporcionando qualidade de vida aos usuários do SUS. (VIEIRA et al., 2017).

A Atenção Básica em Saúde vem sendo a melhor estratégia para combater e acompanhar o tratamento das DCNT, capaz de empregar tecnologias eficazes e interferir não apenas na cura e reabilitação, como também nos fatores determinantes e de controle da saúde individual e coletiva. (ENCARNAÇÃO; SANTOS; HELIOTÉRIO, 2017).

O Hiperdia é um programa implantado pelo Ministério da Saúde definido como uma estratégia de reorganização da atenção à Hipertensão Arterial (HA) e à Diabetes Mellitus (DM), que visa a preparar, capacitar e incentivar os profissionais envolvidos na Atenção Básica à Saúde a promover ações direcionadas ao público, enfatizando a importância da prevenção primária e acompanhamento integral dos usuários diagnosticados de HAS e DM, a fim de promover o controle da doença e garantir uma boa qualidade de vida (VIEIRA et al., 2017).

As Unidades Básicas de Saúde (UBS) recebem as pessoas com diagnósticos de Hipertensão Arterial e Diabetes Mellitus e, por um Programa Estratégia Saúde da Família, passam pela triagem e são acompanhados pelos profissionais da UBS e 
pela equipe multiprofissional, atendendo de forma integrada e com níveis de competência estabelecidos na abordagem da HAS e DM, sendo considerado o primeiro acesso de saúde indicado para as comunidades e, caso seja necessário, os usuários das UBSs serão encaminhados para uma consulta especializada em unidades de referência secundária ou terciária para um atendimento mais complexo (PETERMANN et al., 2015). Nesse sentido, questiona-se sobre a forma de atuação desses programas criados pelo governo. A população está sendo acompanhada e recebe o tratamento que é estabelecido pelo Sistema Único de Saúde (SUS)?

\section{MATERIAIS E MÉTODOS}

A pesquisa foi um estudo exploratório, analítico e descritivo. Realizada na cidade de Cajazeiras, localizada no interior do estado da Paraíba, na qual possui uma população estimada de 61.776 pessoas distribuídos na área territorial de 565 , $899 \mathrm{~km}^{2}$. O local da pesquisa foi uma Unidade Básica de Saúde que fica localizada no bairro Cristo, Cajazeiras - PB, Brasil (IBGE, 2018).

A pesquisa foi realizada entre homens e mulheres, com um total de 30 participantes, todos usuários cadastrados na Unidade Básica de Saúde José Nilson de Sousa, perante a assinatura do Termo de Consentimento Livre e Esclarecido (TCLE). Foram estabelecidos como critérios de inclusão os usuários diagnosticados com Diabetes Mellitus e Hipertensão Arterial Sistêmica, homens e mulheres, com idade média entre 30 e 95 anos. E como critérios de exclusão, os usuários do serviço que apresentassem apenas uma das doenças, gestantes e pessoas abaixo de 30 anos de idade e acima de 95 anos ou que não concordassem com os termos da pesquisa.

O estudo seguiu as normas da resolução n 466/12 do Conselho Nacional de Saúde (CNS) que rege a pesquisa em seres humanos e garante que a privacidade do sujeito da pesquisa seja preservada. Foi submetida ao Comitê de Ética em Pesquisa (CEP) da Faculdade Santa Maria (FSM). Após a expedição do Parecer de número 3.111.129, o estudo iniciou com a coleta no mês de março de 2019, por 
meio de entrevista com os usuários do serviço que se encaixaram nos critérios de inclusão através de um questionário estruturado que apresenta perguntas objetivas no intuito de elencar idade dos pacientes da pesquisa, grau de escolaridade e qual(is) o(s) serviço(s) de atendimentos realizados na Unidade Básica de Saúde e, em seguida, foi executada a análise dos dados.

Os dados obtidos foram analisados estatisticamente, realizando codificação, tabulação e apresentação em forma de tabelas e gráficos com as respectivas distribuições percentuais, através do software Microsoft Office Excel®. A discussão está pautada de acordo com a revisão bibliográfica para subsidiar e fundamentar a pesquisa.

\section{RESULTADOS E DISCUSSÕES}

O presente estudo foi realizado na Unidade Básica de Saúde José Nilson de Sousa, situada na zona urbana na cidade de Cajazeiras-PB, contando com 30 participantes com idade de 34 a 95 anos. Mediante a análise, foi observado que seis (20\%) participantes eram solteiros, 15 (50\%) eram casados, e nove (30\%), viúvos. Quanto ao nível de escolaridade, três (10\%) sem escolaridade, Ensino Fundamental Incompleto com o total de 21 (70\%), Ensino Fundamental com três (10\%) e Ensino Médio com três (10\%) participantes. Em relação à renda familiar, 15 (50\%) entrevistados recebem um salário mínimo mensal, 14 (47\%), dois salários mínimos e um $(3 \%)$, mais de dois salários mínimos.

As patologias HAS e DM desenvolvem-se por diversos fatores, como idade, sexo, raça, histórico familiar, ingestão alimentar, uso de sódio, obesidade, tabagismo e uso de anticoncepcionais orais (SOUSA et al., 2015). Além de desigualdade social, baixa escolaridade, pouco acesso à informação e aos serviços e outros fatores comportamentais como sedentarismo, o transtorno do sono, o mau gerenciamento do estresse, ansiedade e depressão (LOPES; REZENDE; CLABRIA, 2017). 
Tabela 1. Perfil socioeconômico dos usuários cadastrados na Unidade Básica de Saúde - José Nilson de Sousa em Cajazeiras-PB

\begin{tabular}{lcc}
\hline Estado civil & Frequência & Porcentagem \\
\hline Solteiro(as) & 6 & $20 \%$ \\
Casados(as) & 15 & $50 \%$ \\
Viúvos(as) & 9 & $30 \%$ \\
Total & 30 & $100 \%$ \\
\hline Nível de escolaridade & Frequência & Porcentagem \\
Sem Escolaridade & 3 & $10 \%$ \\
Ensino Fundamental Incompleto & 21 & $70 \%$ \\
Ensino Fundamental & 3 & $10 \%$ \\
Ensino Médio & 3 & $10 \%$ \\
Total & 30 & $100 \%$ \\
\hline Renda familiar & Frequência & Porcentagem \\
\hline 1 salário mínimo & 15 & $50 \%$ \\
2 salários mínimos & 14 & $47 \%$ \\
Mais de 2 salários mínimos & 1 & $3 \%$ \\
Total & 30 & $100 \%$
\end{tabular}

Fonte: Própria

Os gráficos 1 e 2 mostram que houve prevalência na pessoa idosa, com predominância do sexo feminino de 73\%, isso é explicado por Freitas et al. (2018), quando relatam que as mulheres são mais interessadas em obter conhecimento das doenças, estão mais atentas aos sintomas e buscam com mais frequência os serviços de saúde, sendo diagnosticadas precocemente. E entre o sexo masculino, o gráfico aponta o percentual de $27 \%$.

Conforme os dados estatísticos do Sistema de Vigilância de Fatores de Risco e Proteção para Doenças Crônicas por Inquérito Telefônico (VIGITEL), do Ministério da Saúde, a prevalência de hipertensão passou de 22,6\% em 2006 para 24,3\% em 2017. O Diabetes Mellitus também está entre umas das DCNT de maior impacto mundial como problema de saúde pública. Entre 2006 e 2016, o número de brasileiros com DM aumentou $61,8 \%$, passando a atingir $5,5 \%$ da população, e dados atuais apontam para $8,9 \%$ das pessoas. $O$ índice mostra que o aumento pode ser em decorrência da qualidade de vida das pessoas que passam a viver mais 
tempo, pois, segundo estudos, a pressão tende a aumentar com a idade, chegando a 60,9\% entre adultos com 65 anos. Quando comparada ao sexo a maior prevalência para o diagnóstico médico de HAS, são constatados $26,4 \%$ do sexo feminino contra $21,7 \%$ para o sexo masculino, e no diagnóstico DM, o índice é de $9 \%$ para o sexo feminino e $7,8 \%$ para o sexo masculino (VIGITEL, 2016).

Gráfico 1: Porcentagem de idade entre os usuários da Unidade Básica de Saúde.

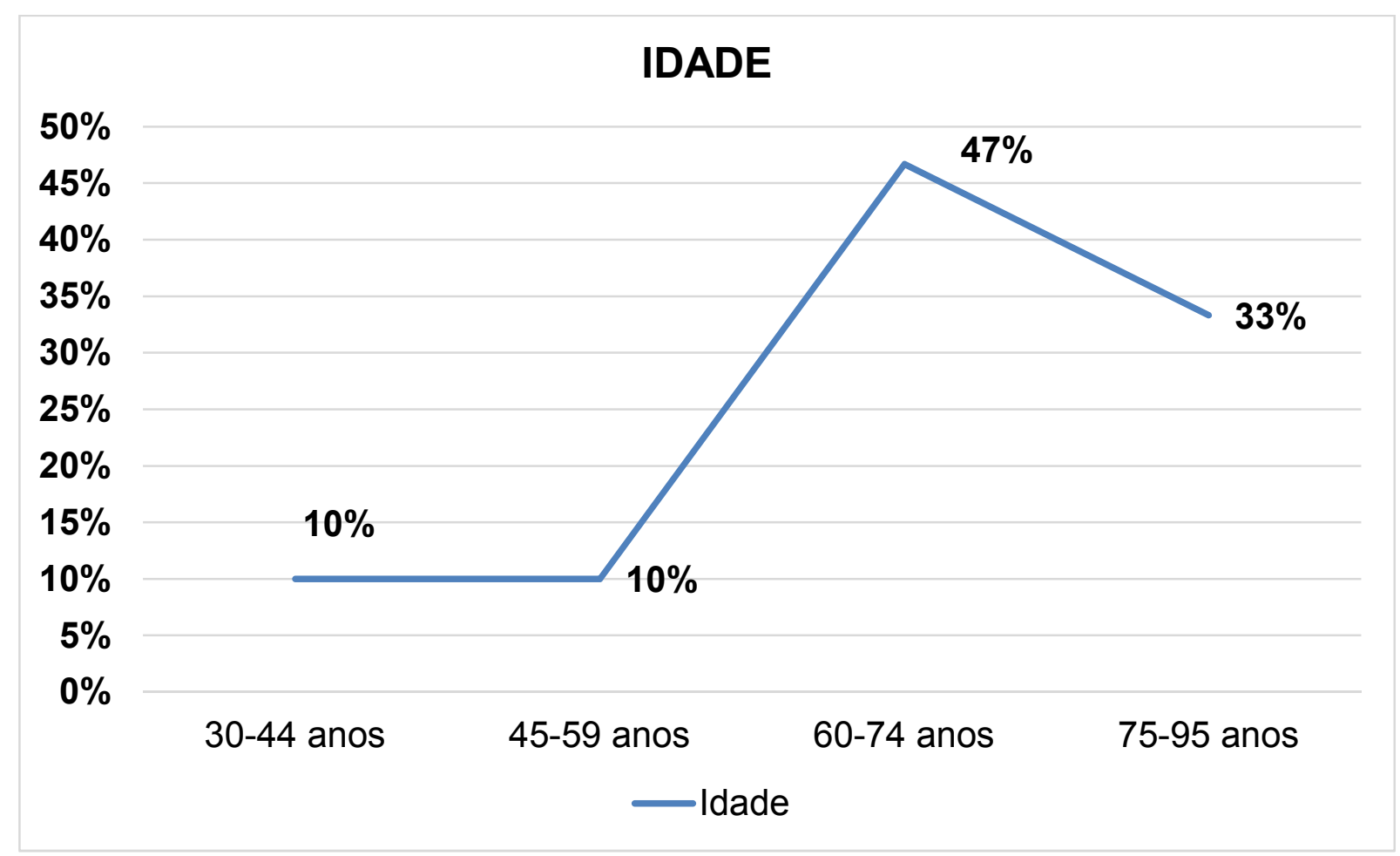

Fonte: Própria. 
Gráfico 2: Relação entre o sexo masculino e Feminino.

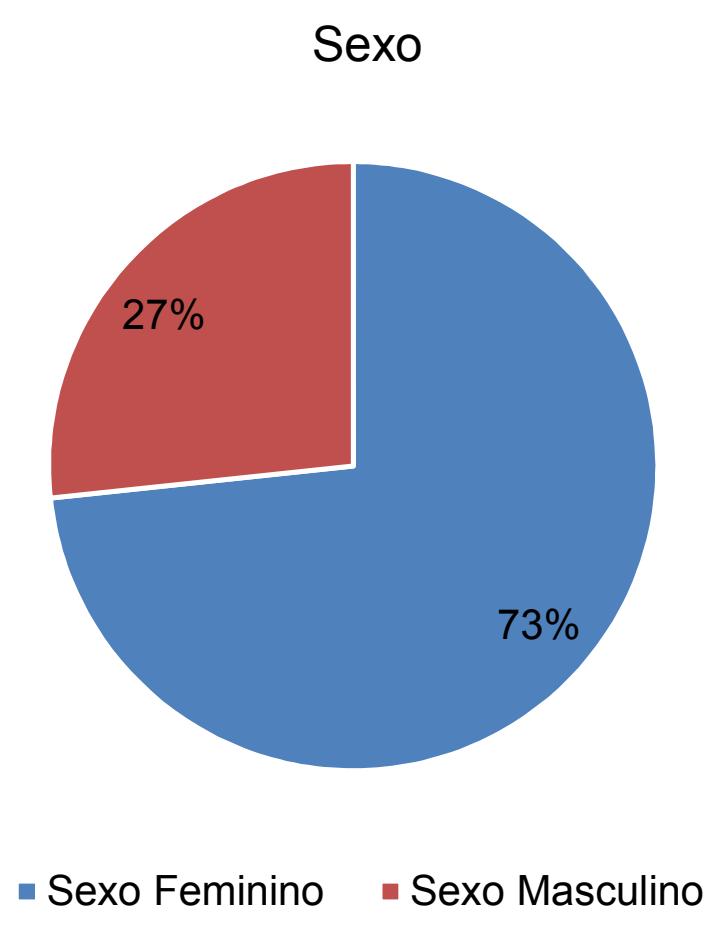

Fonte: Própria.

O gráfico 3 mostra a frequência com que os entrevistados realizam esses exames. Em 2019, para o grupo de 30 participantes, 10 (33\%) realizaram exames de urina, $10(33 \%)$ de creatinina, $10(33 \%)$ de potássio sérico, $10(33 \%)$ de glicemia sérica, $10(33 \%)$ de colesterol total, $10(33 \%)$ de eletrocardiograma de repouso, 10 (33\%) de $\mathrm{Na}$ (sódio), 10 (33\%) de cetonúria, 10 (33\%) de proteína sérica glicada. No ano de 2018, 11 (37\%) pessoas realizaram exames de urina, $11(37 \%)$ de creatinina, $11(37 \%)$ de potássio sérico, $11(37 \%)$ de glicemia sérica, $11(37 \%)$ de colesterol total, 11 (37\%) eletrocardiograma de repouso, 11 (37\%) Na (sódio), 11 (37\%) de cetonúria, $11(37 \%)$ de proteína sérica glicada. Houve relatos de realização de exames há mais de dois anos, com o total de sete $(23 \%)$ que realizaram exames de urina, sete $(23 \%)$ de creatinina, sete $(23 \%)$ de potássio sérico, sete $(23 \%)$ de glicemia sérica, sete $(23 \%)$ de colesterol total, sete $(23 \%)$ de eletrocardiograma de repouso, sete $(23 \%)$ de $\mathrm{Na}$ (sódio), sete $(23 \%)$ de cetonúria, sete $(23 \%)$ de proteína sérica glicada. Dois $(7 \%)$ entrevistados informaram que nunca realizaram exames laboratoriais. 
Algumas complicações do Diabetes Mellitus e da Hipertensão Arterial podem ser controladas e prevenidas através do autocuidado e um acompanhamento adequado dos níveis de colesterol, controle glicêmico e pressão arterial (BERTONHI; DIAS, 2018).

Conforme o protocolo do Ministério da Saúde para o controle dos diagnósticos da HA e DM, aponta-se a realização de serviços auxiliares ao diagnóstico periodicamente para a detecção de uma possível complicação das referidas doenças, considerando os sintomas, resultados de análises glicêmicas, estilo de vida, Índice de Massa Corporal (IMC), verificação da pressão arterial, análise na função renal, exame oral, nível sérico de creatinina, eletrocardiograma, sendo importante uma abordagem complementar de oftalmologista (GONÇALVES; REIS; CARVALHO, 2017).

Gráfico 3: Descrição e frequência de exames realizados pelos entrevistados.

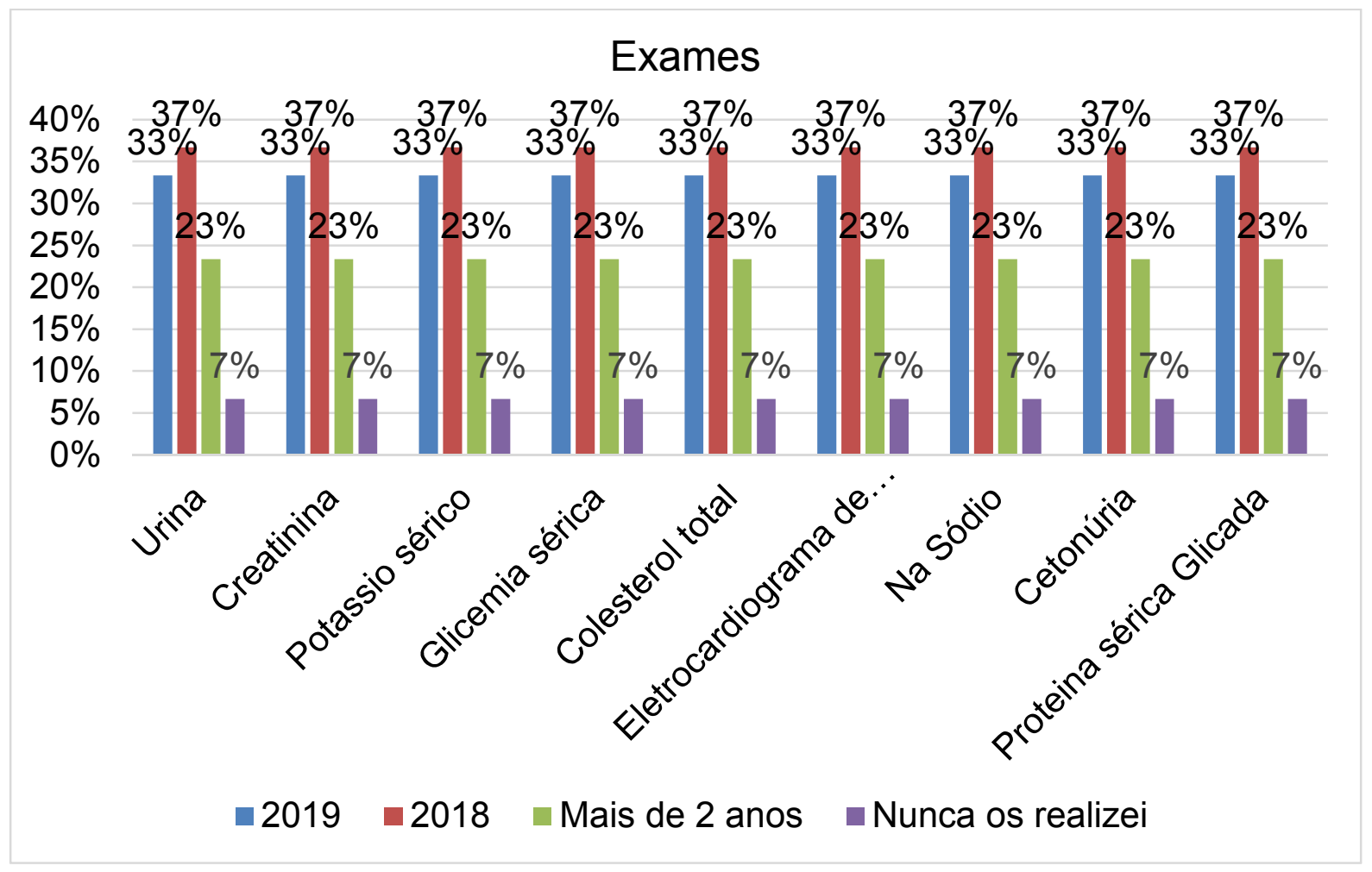

Fonte: Própria.

O gráfico 4 apresenta o percentual de entrevistados que foram encaminhados para outros profissionais de saúde. Das 30 pessoas entrevistadas, nenhum $(0 \%)$ foi 
encaminhado ao Assistente Social, 11 (37\%), ao Cardiologista, 0 (0\%), ao Educador Físico, um (3\%), ao Endocrinologista, Cardiologista e Nefrologista, um (3\%), ao Nefrologista, Cardiologista, Endocrinologista e Nutricionista, quatro (13\%), ao Nutricionista e Cardiologista, dois (7\%), ao Endocrinologista e Cardiologista, um (3\%), ao Psicólogo, Cardiologista e Odontólogo, um (3\%), ao Cardiologista, Nutricionista e Psicólogo, dois (7\%), Nutricionista, Cardiologista e Endocrinologista, dois $(7 \%)$, ao Nutricionista e cinco (17\%) não foram encaminhados para outros profissionais e especialidades referidos ou diferentes.

A importância da integração de grupos multiprofissionais com ações assistenciais e educativas propõe eficácia ao tratamento e qualidade de vida aos diabéticos e hipertensos do SUS. Uma vez que é importante a abordagem multidisciplinar na atenção básica, considerando a necessidade de alterações de hábitos, por se tratar de um agravo multifatorial e necessitar de um acompanhamento da equipe a fim de obter uma maior afetividade do tratamento (SOUSA et al., 2015).

De acordo com uma pesquisa de Fernandez et al. (2016), o programa Hiperdia necessita de ações eficazes para gerar mudanças de comportamento dos usuários da HAS e DM, sendo um desafio aos profissionais da equipe profissional de saúde. 
Gráfico 4: Percentual de usuários do serviço encaminhados pelo médico ou enfermeira da Unidade Básica de Saúde a profissionais da equipe multidisciplinar ou especialistas.

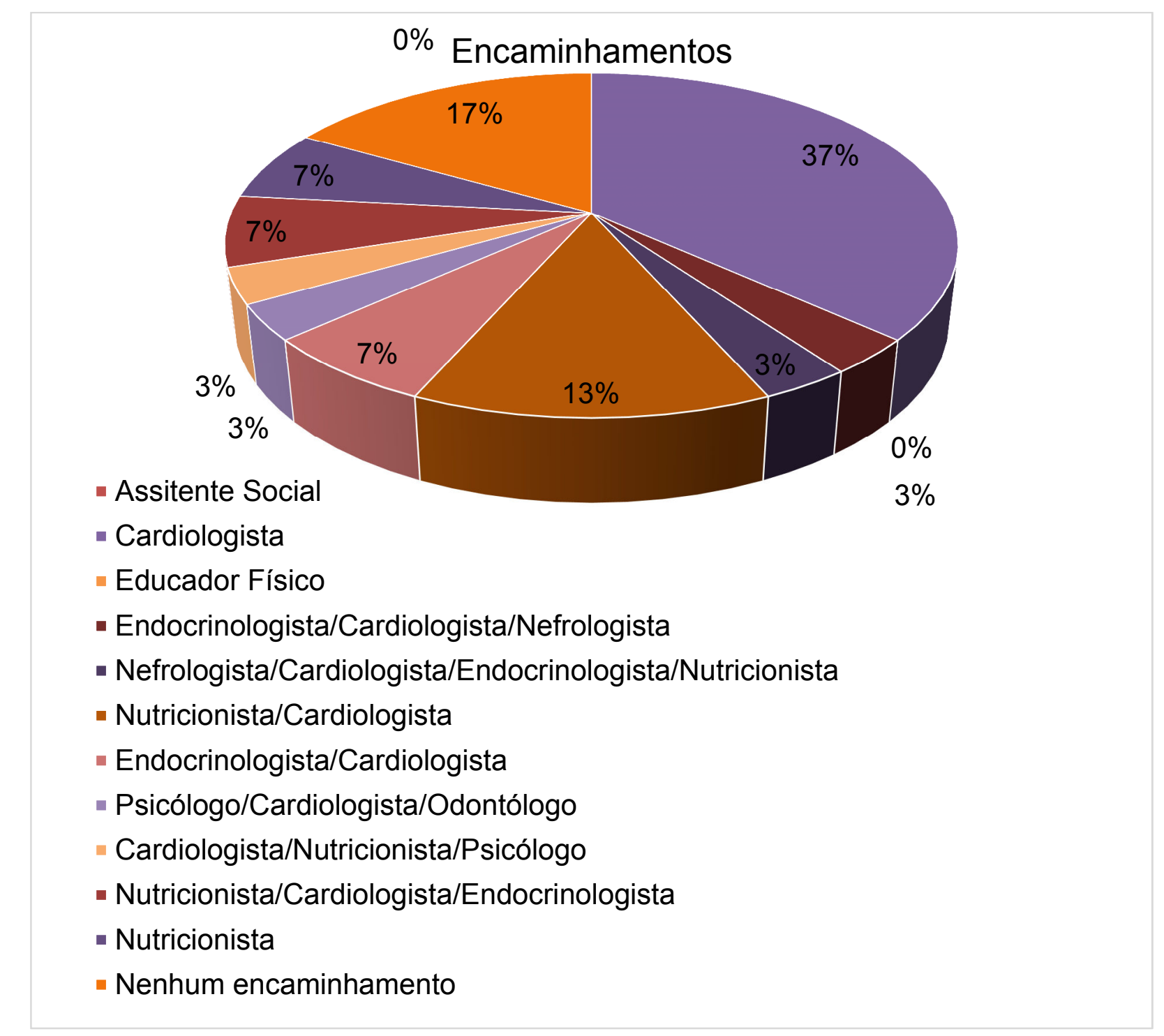

Fonte: Própria.

\section{CONCLUSÃO}

De acordo com as informações coletadas, foi possível mostrar que, no perfil dos usuários do SUS diagnosticados por Hipertensão Arterial e Diabetes Mellitus da 
Unidade Básica de Saúde José Nilton de Sousa no município de Cajazeiras, predomina o sexo feminino, com baixo nível de escolaridade e baixa renda salarial, pois a maioria é aposentada ou recebe auxílio assistencial do Governo Federal.

As análises confirmaram que o maior número de participantes não realiza os procedimentos para o acompanhamento de possíveis agravos nas doenças, bem como o uso da farmacoterapia para possível alteração de medicamento, visto que a sua dosagem depende da forma de tratamento ao qual estão submetidos e se há efetivação com eficácia, já que o tratamento dessas doenças crônicas requer uma mudança no estilo de vida.

A pesquisa mostra que as ações desenvolvidas pela UBS não são realizadas de acordo com o Plano de Reorganização da Atenção à Hipertensão Arterial e ao Diabetes Mellitus apresentado pelo Governo Federal através do Ministério da Saúde. Conforme os dados obtidos, observa-se que os usuários do SUS não são instruídos pelo médico/enfermeiro da UBS a procurarem outros profissionais da saúde, como nutricionistas, psicólogo e médicos especialistas, a fim de fazer exames detalhados, promovendo, assim, a prevenção terciária com a finalidade de prevenir ou retardar o desenvolvimento de complicações agudas e crônicas da HAS e DM e a morte.

Por fim, o estudo mostra que é necessário uma busca ativa dos profissionais de saúde até os usuários do serviço que são acometidos pelas patologias de HAS e DM, visto que a maioria sente uma dificuldade em se locomover até a UBS por complicações decorrentes da própria patologia, acessibilidade considerando que a Unidade de Saúde é situada em um morro e atende várias comunidades.

\section{REFERÊNCIAS BIBLIOGRÁFICAS}

BERTONHI L, DIAS J.. Diabetes mellitus tipo 2: aspectos clínicos, tratamento e conduta dietoterápica. Revista Ciências Nutricionais online, v.2, n.2, p.1-10, jan, 2018.

BRASIL. Ministério da Saúde. Secretaria de Atenção à Saúde. Departamento de Atenção Básica. Estratégias para o cuidado da pessoa com doença crônica: hipertensão arterial sistêmica / Ministério da Saúde, Secretaria de Atenção à Saúde, Departamento de Atenção Básica. - Brasília: Ministério da Saúde, 2013. 128 p.: il. (Cadernos de Atenção Básica, n. 37).

CORTEZ D, et al. Complicações e o tempo de diagnóstico do diabetes mellitus na atenção primaria. Acta Paul Enferm.: v.28, n.3, p.250-251. Jan, 2015.

ENCARNAÇÃO P, SANTOS E, HELIOTÉRIO M. Consulta de enfermagem para pessoas com diabetes e hipertensão na atenção básica: um relato de experiência. Rev. APS. v.20, n.2, 
p.275. abr/jun, 2017.

FERNANDEZ D, POLLARO S, GONÇALVES L. Programa hiperdia e suas repercussões sobre os usuários. Revista Baiana de Enfermagem, Salvador, v 30, n.3, p.2, jul./set.2016.

FREITAS P, et al. Uso de serviços de saúde e de medicamentos por portadores e Hipertensão e Diabetes no Município do Rio de Janeiro. Brasil. Ciência \& Saúde Coletiva, v.23, n.7, p.2384, 2388, 2389. jan, 2018.

GONÇALVES P, REIS J, CARVALHO R. Diabetes Mellitus e fatores externos que acarretam esta disfunção. II Congresso Brasileiro de Ciências de Saúde, p.2, 2017.

GOVERNO DO BRASIL. Número de brasileiros com diabetes cresceu $61,8 \%$ em 10 anos. Disponivel em: http://portalarquivos.saude.gov.br/images/pdf/2017/abril/17/Vigitel.pdf Acesso em: 16 de outubro de 2018.

LOPES P, REZENDE A, CLABRIA L. Fatores de risco para Doenças Crônicas Não Transmissíveis em universitários. Revista Brasileira em Promoção da Saúde, Fortaleza, v.30, n.4, p.2-11, out./dez., 2017.

PEREIRA M, FIGUEIREDO A. A importância do diagnóstico da Diabetes Mellitus tipos 1 e 2 na infância. SALUSVITA, Bauru, v. 36, n. 2, p. 603-606, maio 2017.

PETERMANN X, et al. Epidemiologia e cuidado à Diabetes Mellitus praticado na Atenção Primaria à Saúde: uma revisão narrativa. Saúde (Santa Maria). Santa Maria, Vol. 41, n. 1, p.456, Jan./Jul, 2015.

SOUSA A, et al. Cuidados de enfermagem ao cliente hipertenso na estratégia saúde da família. Rev. Enfermagem UERJ, v. 23, n.1, p.103. Rio de Janeiro, jan/fev 2015.

VIEIRA V, et al. Cuidados de enfermagem para pessoas com diabetes mellitus e hipertensão arterial: mapeamento cruzado. Revista baiana de enfermagem; v.31, n.4, p.2, jan., 2017. 